\title{
Existence, uniqueness and almost surely asymptotic estimations of the solutions to neutral stochastic functional differential equations driven by pure jumps
}

\author{
Wei $\mathrm{Mao}^{1}$,Quanxin Zhu ${ }^{2}$, Xuerong $\mathrm{Mao}^{3}$ \\ 1.School of Mathematics and Information Technology, Jiangsu Second Normal \\ University, Nanjing, 210013, Jiangsu, P.R.China; \\ 2.School of Mathematical Sciences, Nanjing Normal University, Nanjing, 210023, \\ Jiangsu, P.R.China; \\ 3.Department of Mathematics and Statistics, University of Strathclyde, Glasgow, G1 \\ $1 X H, U K$.
}

\begin{abstract}
In this paper, we are concerned with neutral stochastic functional differential equations driven by pure jumps (NSFDEwPJs). We prove the existence and uniqueness of the solution to NSFDEwPJs whose coefficients satisfying the Local Lipschitz condition. In addition, we establish the $p$-th exponential estimations and almost surely asymptotic estimations of the solution for NSFDEwJs.
\end{abstract}

Key words: Neutral stochastic functional differential equations, Pure jumps, Existence and uniqueness, Exponential estimations, Almost surely asymptotic estimations.

\section{Introduction}

Stochastic delay differential equations (SDDEs) have come to play an important role in many branches of science and industry. Such models have been used with great success in a variety of application areas, including biology, epidemiology, mechanics, economics and finance. In the past few decades, qualitative theory of SDDEs have been studied intensively by many scholars. Here, we refer to S.E.A.Mohammed [1], X.Mao [2-5,9], E.Buckwar [6], U.Kuchler [7], Y.Hu [8], D.Xu [10], F.Wu [11], J.Appleby [12], I.Gyongy [13] and references therein. Recently, motivated by the theory of aeroelasticity, a class of neutral stochastic equations has also received a great deal of 
attention and much work has been done on neutral stochastic equations. For example, conditions of the existence and stability of the analytical solution are given in [14-20]. Various efficient computational methods are obtained and their convergence and stability have been studied in [21-25].

However, all equations of the aboved mentioned works are driven by white noise perturbations with continuous initial data and white noise perturbations are not always appropriate to interpret real datas in a reasonable way. In real phenomena, the state of neutral stochastic delay equations may be perturbed by abrupt pulses or extreme events. A more natural mathematical framework for these phenomena has been taken into account other than purely Brownian perturbations. In particular, we incorporate the Levy perturbations with jumps into neutral stochastic delay equations to model abrupt changes.

In this paper, we study the following neutral stochastic functional differential equations with pure jumps (NSFDEwPJs)

$$
d\left[x(t)-D\left(x_{t}\right)\right]=f\left(x_{t}, t\right) d t+\int_{U} h\left(x_{t}, u\right) N_{\bar{p}}(d t, d u), \quad t_{0} \leq t \leq T .
$$

To the best of our knowledge, there are no literatures concerned with the existence and asymptotic estimations of the solution to NSFDEwPJs (1). On the one hand, we prove that equation (1) has a unique solution in the sense of $L^{P}$ norm. We don't use the fixed point Theorem. Instead, we get the solution of equation (1) via successive approximations. On the other hand, we study the $p$-th exponential estimations and almost surely asymptotic estimations of the solution to equation (1). By using the It $\widehat{o}$ formula, Taylor formula and the Burkholder Davis inequality, we have that the $p$-th moment of the solution will grow at most exponentially with exponent $M$ and show that the exponential estimations implies almost surely asymptotic estimations. Although the way of analysis follows the ideas in [2], however, those results on the existence and uniqueness of the solution in [2] can not be extended to the jumps case naturally. Unlike the Brown process whose almost all sample paths are continuous, the Poisson random measure $N_{\bar{p}}(d t, d u)$ is a jump process and has the sample paths which are right-continuous and have left limits. Therefore, there is a great difference between the stochastic integral with respect to the Brown process and the one with respect to the Poisson random measure. It should be pointed out that the proof for NSFDEwPJs is certainly not a straightforward generalization of that for NSFDEs without 
jumps and some new techniques are developed to cope with the difficulties due to the Poisson random measures.

The rest of the paper is organized as follows. In Section 2, we introduce some notations and hypotheses concerning equation (1); In Section 3, the existence and uniqueness of the solution to equation (1) are investigated; In Section 4, we prove the $p$-th moment of the solution will grow at most exponentially with exponent $M$ and show that the exponential estimations implies the almost surely asymptotic estimations.

\section{Preliminaries}

Let $(\Omega, \mathcal{F}, P)$ be a complete probability space equipped with some filtration $\left(\mathcal{F}_{t}\right)_{t \geq t_{0}}$ satisfying the usual conditions, (i.e. it is right continuous and $\left(\mathcal{F}_{t_{0}}\right)$ contains all $P$-null sets $)$. Let $\tau>0$, and $D\left([-\tau, 0] ; R^{n}\right)$ denote the family of all right-continuous functions with left-hand limits $\varphi$ from $[-\tau, 0] \rightarrow R^{n}$. The space $D\left([-\tau, 0] ; R^{n}\right)$ is assumed to be equipped with the norm $\|\varphi\|=$ $\sup _{-\tau \leq t \leq 0}|\varphi(t)|$ and $|x|=\sqrt{x^{\top} x}$ for any $x \in R^{n}$. If $A$ is a vector or matrix,

its trace norm is denoted by $|A|=\sqrt{\operatorname{trace}\left(A^{\top} A\right)}$, while its operator norm is denoted by $\|A\|=\sup \{|A x|:|x|=1\}$. $D_{\mathcal{F}_{0}}^{b}\left([-\tau, 0] ; R^{n}\right)$ denotes the family of all almost surely bounded, $\mathcal{F}_{0}$-measurable, $D\left([-\tau, 0] ; R^{n}\right)$ valued random variable $\xi=\{\xi(\theta):-\tau \leq \theta \leq 0\}$. Let $t_{0} \geq 0, p \geq 2, \mathcal{L}_{\mathcal{F}_{t_{0}}}^{p}\left([-\tau, 0] ; R^{n}\right)$ denote the family of all $\mathcal{F}_{t_{0}}$ measurable, $D\left([-\tau, 0] ; R^{n}\right)$-valued random variables $\varphi=\{\varphi(\theta):-\tau \leq \theta \leq 0\}$ such that $\mathrm{E} \sup _{-\tau \leq \theta \leq 0}|\varphi(\theta)|^{p}<\infty$.

Let $(U, \mathcal{B}(U))$ be a measurable space and $\pi(d u)$ a $\sigma$ - finite measure on it. Let $\left\{\bar{p}=\bar{p}(t), t \geq t_{0}\right\}$ be a stationary $\mathcal{F}_{t}$-Poisson point process on $U$ with a characteristic measure $\pi$. Then, for $A \in \mathcal{B}(U-\{0\})$, here $0 \in$ the closure of $A$, the Poisson counting measure $N_{\bar{p}}$ is defined by

$$
N_{\bar{p}}\left(\left(t_{0}, t\right] \times A\right):=\sharp\left\{t_{0}<s \leq t, \bar{p}(s) \in A\right\}=\sum_{t_{0}<s \leq t} I_{A}(\bar{p}(s)),
$$

where $\sharp$ denotes the cardinality of a set. For simplicity, we denote: $N_{\bar{p}}(t, A):=$ $N_{\bar{p}}\left(\left(t_{0}, t\right] \times A\right)$. It follows from [26] that there exists a $\sigma$ - finite measure $\pi$ satisfying

$$
E\left[N_{\bar{p}}(t, A)\right]=\pi(A) t, \quad P\left(N_{\bar{p}}(t, A)=n\right)=\frac{e(-t \pi(A))(\pi(A) t)^{n}}{n !} .
$$


This measure $\pi$ is called the Levy measure. Then, the measure $\tilde{N}_{\bar{p}}$ is defined by

$$
\tilde{N}_{\bar{p}}\left(\left[t_{0}, t\right], A\right):=N_{\bar{p}}\left(\left[t_{0}, t\right], A\right)-t \pi(A), \quad t>t_{0} .
$$

We refer to N.Ikeda [26] for the details on Poisson point process.

The integral version of equation (1) is given by the equation

$$
x(t)-D\left(x_{t}\right)=x_{t_{0}}-D\left(x_{t_{0}}\right)+\int_{t_{0}}^{t} f\left(x_{s}, s\right) d s+\int_{t_{0}}^{t} \int_{U} h\left(x_{s}, u\right) N_{\bar{p}}(d s, d u),
$$

where

$$
x_{t}=\{x(t+\theta):-\tau \leq \theta \leq 0\}
$$

is regarded as a $D\left([-\tau, 0] ; R^{n}\right)$-valued stochastic process. $f: D\left([-\tau, 0] ; R^{n}\right) \times$ $\left[t_{0}, T\right] \rightarrow R^{n}$ and $h: D\left([-\tau, 0] ; R^{n}\right) \times U \rightarrow R^{n}$ are both Borel-measurable functions. The initial condition $x_{t_{0}}$ is defined by

$$
x_{t_{0}}=\xi=\{\xi(t):-\tau \leq t \leq 0\} \in \mathcal{L}_{\mathcal{F}_{t_{0}}}^{p}\left([-\tau, 0] ; R^{n}\right),
$$

that is, $\xi$ is an $\mathcal{F}_{t_{0}}$-measurable $D\left([-\tau, 0] ; R^{n}\right)$-valued random variable and $E\|\xi\|^{p}<\infty$. $\tilde{N}_{\bar{p}}(d t, d u)$ is the compensated Poisson random measure given by

$$
\tilde{N}_{\bar{p}}(d t, d u)=N_{\bar{p}}(d t, d u)-\pi(d u) d t
$$

here $\pi(d u)$ is the Levy measure associated to $N_{\bar{p}}$.

To study the existence and asymptotic estimations of the solution to equation (1), we consider the following hypotheses.

(H1) Let $D(0)=0$ and for all $\varphi, \psi \in D\left([-\tau, 0] ; R^{n}\right)$, there exists a constant $k_{0} \in(0,1)$ such that

$$
|D(\varphi)-D(\psi)| \leq k_{0}\|\varphi-\psi\| .
$$

(H2) For all $\varphi, \psi \in D\left([-\tau, 0] ; R^{n}\right), t \in\left[t_{0}, T\right]$ and $u \in U$, there exist two positive constants $k$ and $L_{0}$ such that

$$
\begin{gathered}
|f(\varphi, t)-f(\psi, t)|^{2} \vee \int_{U}|h(\varphi, u)-h(\psi, u)|^{2} \pi(d u) \leq k\|\varphi-\psi\|^{2} \\
|f(0, t)|^{2} \vee|h(0, u)|^{2} \leq L_{0}
\end{gathered}
$$


(H3) For all $\varphi, \psi \in D\left([-\tau, 0] ; R^{n}\right), p \geq 2$ and $u \in U$, there exists a positive constant $L$ such that

$$
|h(\varphi, u)-h(\psi, u)|^{p} \leq L \| \varphi-\psi||^{p}|u|^{p}
$$

where $\pi(U)<\infty$ and $\int_{U}|u|^{p} d u<\infty$.

Clearly, (H2) and (H3) implies the linear growth condition

$$
|f(\varphi, t)|^{2} \vee \int_{U}|h(\varphi, u)|^{2} \pi(d u) \leq L_{1}\left(1+\|\varphi\|^{2}\right),
$$

and

$$
\int_{U}|h(\varphi, u)|^{p} \pi(d u) \leq L_{2}\left(1+\|\varphi\|^{p}\right)
$$

where $L_{1}$ and $L_{2}$ are two positive constants.

In fact, for any $\varphi \in D\left([-\tau, 0] ; R^{n}\right)$ and $t \in\left[t_{0}, T\right]$, it follows from (4) and (5) that

$$
\begin{aligned}
|f(\varphi, t)|^{2} & \leq 2\left[|f(\varphi, t)-f(0, t)|^{2}+|f(0, t)|^{2}\right] \\
& \leq 2\left(k\|\varphi\|^{2}+L_{0}\right) \leq L_{1}\left(1+\|\varphi\|^{2}\right) .
\end{aligned}
$$

and

$$
\begin{aligned}
\int_{U}|h(\varphi, u)|^{2} \pi(d u) & \leq 2\left[\int_{U}|h(\varphi, u)-h(0, u)|^{2} \pi(d u)+\int_{U}|h(0, u)|^{2} \pi(d u)\right] \\
& \leq 2\left(k\|\varphi\|^{2}+2 L_{0} \pi(U)\right) \leq L_{1}\left(1+\|\varphi\|^{2}\right) .
\end{aligned}
$$

where $L_{1}=\max \left\{2 k, 2 L_{0}, 2 L_{0} \pi(U)\right\}$. Similarly, for any $\varphi \in D\left([-\tau, 0] ; R^{n}\right)$ and $t \in\left[t_{0}, T\right]$, it follows from (5) and (6) that

$$
\begin{aligned}
\int_{U}|h(\varphi, u)|^{p} \pi(d u) & \leq 2^{p-1} \int_{U}\left(|h(\varphi, u)-h(0, u)|^{p}+|h(0, u)|^{p}\right) \pi(d u) \\
& \leq 2^{p-1} \int_{U}|u|^{p} d u k_{1}|| \varphi \|^{p}+2^{p-1} L_{0}^{\frac{p}{2}} \pi(U) \leq L_{2}\left(1+\| \varphi||^{p}\right),
\end{aligned}
$$

where $L_{2}=\max \left\{2^{p-1} \int_{U}|u|^{p} d u k_{1}, 2^{p-1} L_{0}^{\frac{p}{2}} \pi(U)\right\}$. Hence, the linear growth conditions (7) and (8) are satisfied.

Now we present the definition of the solution to equation (1). 
Definition 2.1 A right continuous with left limits process $x=\{x(t), t \in$ $\left.\left[t_{0}, T\right]\right\}\left(t_{0}<T<\infty\right)$ is called a solution of equation (1) if

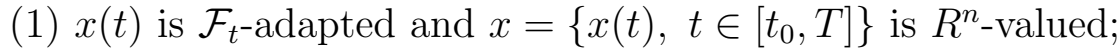

(2) $\int_{t_{0}}^{T}|x(t)|^{2} d s<\infty$, a.s.;

(3) $x(t)=\xi$ and, for every $t_{0} \leq t \leq T$,

$x(t)-D\left(x_{t}\right)=x_{t_{0}}-D\left(x_{t_{0}}\right)+\int_{t_{0}}^{t} f\left(x_{s}, s\right) d s+\int_{t_{0}}^{t} \int_{U} h\left(x_{s}, u\right) N_{\bar{p}}(d s, d u)$ a.s.

A solution $x(t)$ is said to be unique if any other solution $y(t)$ is indistinguishable from it, that is,

$$
P\left\{x(t)=y(t), t \in\left[t_{0}, T\right]\right\}=1 .
$$

\section{The existence and uniqueness theorem}

In this section, we establish the existence and uniqueness of the solution to equation (1) under the Lipschitz condition and the local Lipschitz condition.

Define $x_{t_{0}}^{0}=\xi$ and $x^{0}(t)=\xi(0)$ for $t \in\left[t_{0}, T\right]$. Let $x_{t_{0}}^{n}=\xi, n=1,2, \cdots$ and define the sequence of successive approximations to equation (1)

$$
\begin{aligned}
x^{n}(t)-D\left(x_{t}^{n}\right)= & \xi(0)-D(\xi)+\int_{t_{0}}^{t} f\left(x_{s}^{n-1}, s\right) d s \\
& +\int_{t_{0}}^{t} \int_{U} h\left(x_{s}^{n-1}, u\right) N_{\bar{p}}(d s, d u), n \geq 1 .
\end{aligned}
$$

Theorem 3.1 Let $p \geq 2$ and suppose that the coefficients of equation (1) satisfy conditions (H1)-(H3), then equation (1) has a unique solution $x(t)$ on $\left[t_{0}, T\right]$ in the sense of $L^{p}$-norm.

In order to prove this theorem, let us present three useful lemmas.

Lemma 3.1 [2] Let $p \geq 2, \varepsilon>0$ and $a, b \in R$, then

$$
|a+b|^{p} \leq\left[1+\varepsilon^{\frac{1}{p-1}}\right]^{p-1}\left(|a|^{p}+\frac{|b|^{p}}{\varepsilon}\right) .
$$

Lemma 3.2 Under conditions (H1)-(H3), there exists a positive constant $c$ such that

$$
E \sup _{t_{0} \leq t \leq T}\left|x^{n}(t)\right|^{p} \leq c
$$


where $c=\left(c_{3}+c_{4}\left(T-t_{0}\right) E\|\xi\|^{p}\right) e^{c_{4}\left(T-t_{0}\right)}, c_{3}$ and $c_{4}$ are two positive constants of (25).

Proof: For any $\varepsilon>0$, it follows from Lemma 3.1 that

$$
\begin{aligned}
\left|x^{n}(t)\right|^{p} & =\left|D\left(x_{t}^{n}\right)+x^{n}(t)-D\left(x_{t}^{n}\right)\right| \\
& \leq\left[1+\varepsilon^{\frac{1}{p-1}}\right]^{p-1}\left(\left|x^{n}(t)-D\left(x_{t}^{n}\right)\right|^{p}+\frac{\left|D\left(x_{t}^{n}\right)\right|^{p}}{\varepsilon}\right) .
\end{aligned}
$$

By (H1), one gets

$$
\left|x^{n}(t)\right|^{p} \leq\left[1+\varepsilon^{\frac{1}{p-1}}\right]^{p-1}\left(\left|x^{n}(t)-D\left(x_{t}^{n}\right)\right|^{p}+\frac{\left.k_{0}^{p}\left\|x_{t}^{n}\right\|\right|^{p}}{\varepsilon}\right) .
$$

Letting $\varepsilon=\left[\frac{k_{0}}{1-k_{0}}\right]^{p-1}$ and taking the expectation on both sides of (13), we have

$$
\begin{aligned}
E \sup _{t_{0} \leq s \leq t}\left|x^{n}(s)\right|^{p} \leq & k_{0} E \sup _{t_{0} \leq s \leq t}\left\|x_{s}^{n}\right\|^{p} \\
& +\frac{1}{\left(1-k_{0}\right)^{p-1}} E \sup _{t_{0} \leq s \leq t}\left|x^{n}(s)-D\left(x_{s}^{n}\right)\right|^{p} .
\end{aligned}
$$

On the other hand, we have

$$
\begin{aligned}
E \sup _{t_{0} \leq s \leq t}\left\|x_{s}^{n}\right\|^{p} & \leq E \sup _{t_{0}-\tau \leq s \leq t}\left|x^{n}(s)\right|^{p} \\
& \leq E \| \xi||^{p}+E \sup _{t_{0} \leq s \leq t}\left|x^{n}(s)\right|^{p} .
\end{aligned}
$$

Combing (14) and (15), we obtain

$$
E \sup _{t_{0} \leq s \leq t}\left|x^{n}(s)\right|^{p} \leq \frac{k_{0}}{1-k_{0}} E\|\xi\|^{p}+\frac{1}{\left(1-k_{0}\right)^{p}} E \sup _{t_{0} \leq s \leq t}\left|x^{n}(s)-D\left(x_{s}^{n}\right)\right|^{p} .
$$

By (9) and using the inequality $|a+b+c|^{p} \leq 3^{p-1}\left[|a|^{p}+|b|^{p}+|c|^{p}\right]$, we have

$$
\begin{aligned}
& E \sup _{t_{0} \leq s \leq t}\left|x^{n}(s)-D\left(x_{s}^{n}\right)\right|^{p} \\
\leq & 3^{p-1}\left(E\left(\sup _{t_{0} \leq s \leq t}|\xi(0)-D(\xi)|^{p}\right)+E \sup _{t_{0} \leq s \leq t}\left|\int_{t_{0}}^{s} f\left(x_{\sigma}^{n-1}, \sigma\right) d \sigma\right|^{p}\right. \\
& \left.+E \sup _{t_{0} \leq s \leq t}\left|\int_{t_{0}}^{s} \int_{U} h\left(x_{\sigma}^{n-1}, u\right) N_{\bar{p}}(d \sigma, d u)\right|^{p}\right) \\
:= & 3^{p-1} \sum_{i=1}^{3} I_{i} .
\end{aligned}
$$


Let us estimate the terms introduced above. Letting $\varepsilon=k_{0}^{p-1}$, then it follows from Lemma 3.1 that

$$
I_{1} \leq\left(1+k_{0}\right)^{p} E|||\xi|^{p}
$$

By using the Hölder inequality and the linear growth condition (7), one gets

$$
\begin{aligned}
I_{2} & \leq\left(t-t_{0}\right)^{p-1} E \int_{t_{0}}^{t}\left|f\left(x_{s}^{n-1}, s\right)\right|^{p} d s \\
& \leq\left(t-t_{0}\right)^{p-1} E \int_{t_{0}}^{t}\left[L_{1}\left(1+\left\|x_{s}^{n-1}\right\|^{2}\right)^{2}\right]^{\frac{p}{2}} d s \\
& \leq\left(t-t_{0}\right)^{p-1}\left(2 L_{1}\right)^{\frac{p}{2}} E \int_{t_{0}}^{t}\left(1+\left\|x_{s}^{n-1}\right\|^{p}\right) d s .
\end{aligned}
$$

For the third term $I_{3}$ in (17), we have

$$
\begin{aligned}
I_{3}= & E \sup _{t_{0} \leq s \leq t}\left|\int_{t_{0}}^{s} \int_{U} h\left(x_{\sigma}^{n-1}, u\right) \tilde{N}_{\bar{p}}(d \sigma, d u)+\int_{t_{0}}^{s} \int_{U} h\left(x_{\sigma}^{n-1}, u\right) \pi(d u) d \sigma\right|^{p} \\
\leq & 2^{p-1} E \sup _{t_{0} \leq s \leq t}\left|\int_{t_{0}}^{s} \int_{U} h\left(x_{\sigma}^{n-1}, u\right) \tilde{N}_{\bar{p}}(d \sigma, d u)\right|^{p} \\
& +2^{p-1} E \sup _{t_{0} \leq s \leq t}\left|\int_{t_{0}}^{s} \int_{U} h\left(x_{\sigma}^{n-1}, u\right) \pi(d u) d \sigma\right|^{p}
\end{aligned}
$$

where $\tilde{N}_{p}(d t, d u):=N_{p}(d t, d u)-\pi(d u) d t$. For the last term of $(20)$, using the Hölder inequality and condition (7), we obtain

$$
\begin{aligned}
& E \sup _{t_{0} \leq s \leq t}\left|\int_{t_{0}}^{s} \int_{U} h\left(x_{\sigma}^{n-1}, u\right) \pi(d u) d \sigma\right|^{p} \\
\leq & E\left[\int_{t_{0}}^{t} d s\right]^{p-1} \int_{t_{0}}^{t}\left|\int_{U} h\left(x_{s}^{n-1}, u\right) \pi(d u)\right|^{p} d s \\
\leq & \left(t-t_{0}\right)^{p-1} E \int_{t_{0}}^{t}\left[\int_{U} \pi(d u)\right]^{\frac{p}{2}}\left[\int_{U}\left|h\left(x_{s}^{n-1}, u\right)\right|^{2} \pi(d u)\right]^{\frac{p}{2}} d s \\
\leq & \left(t-t_{0}\right)^{p-1}[\pi(U)]^{\frac{p}{2}} E \int_{t_{0}}^{t}\left[L_{1}\left(1+|| x_{s}^{n-1}||^{2}\right)\right]^{\frac{p}{2}} d s \\
\leq & \left(t-t_{0}\right)^{p-1}\left(2 L_{1}\right)^{\frac{p}{2}}[\pi(U)]^{\frac{p}{2}} E \int_{t_{0}}^{t}\left(1+\| x_{s}^{n-1}||^{p}\right) d s .
\end{aligned}
$$


Now let us estimate the martingale part in (20). By the Kunita's estimates (see Kunita [27] and Applebaum [28]), conditions (7)-(8) and properties of stochastic integral with respect to a Poisson random measure, we have a positive real number $c_{p}$ such that the following inequality holds:

$$
\begin{aligned}
& E \sup _{t_{0} \leq s \leq t}\left|\int_{t_{0}}^{s} \int_{U} h\left(x_{\sigma}^{n-1}, u\right) \tilde{N}_{\bar{p}}(d \sigma, d u)\right|^{p} \\
\leq & c_{p}\left\{E \int_{t_{0}}^{t}\left[\int_{U}\left|h\left(x_{s}^{n-1}, u\right)\right|^{2} \pi(d u)\right]^{\frac{p}{2}} d s+E\left[\int_{t_{0}}^{t} \int_{U}\left|h\left(x_{s}^{n-1}, u\right)\right|^{p} \pi(d u) d s\right]\right\} \\
\leq & c_{p}\left\{E \int_{t_{0}}^{t}\left[L_{1}\left(1+\left\|x_{s}^{n-1}\right\|\right)\right]^{\frac{p}{2}} d s+L_{2} E \int_{t_{0}}^{t}\left(1+\left\|x_{s}^{n-1}\right\|^{p}\right) d s\right\} \\
\leq & c_{p}\left[\left(2 L_{1}\right)^{\frac{p}{2}}+L_{2}\right] E \int_{t_{0}}^{t}\left(1+\left\|x_{s}^{n-1}\right\|^{p}\right) d s .
\end{aligned}
$$

Inserting (21) and (22) into (20), we obtain that

$$
I_{3} \leq c_{1} E \int_{t_{0}}^{t}\left(1+\left\|x_{s}^{n-1}\right\|^{p}\right) d s .
$$

where $c_{1}=2^{p-1}\left[\left(2 L_{1}\right)^{\frac{p}{2}}\left(t-t_{0}\right)^{p-1}(\pi(U))^{\frac{p}{2}}+c_{p}\left(\left(2 L_{1}\right)^{\frac{p}{2}}+L_{2}\right)\right]$. Therefore,

$$
\begin{aligned}
& E \sup _{t_{0} \leq s \leq t}\left|x^{n}(s)-D\left(x_{s}^{n}\right)\right|^{p} \\
\leq & 3^{p-1}\left(1+k_{0}\right)^{p} E\|\xi\|^{p}+c_{2} E \int_{t_{0}}^{t}\left(1+\left\|x_{s}^{n-1}\right\|^{p}\right) d s,
\end{aligned}
$$

where $c_{2}=3^{p-1}\left[\left(t-t_{0}\right)^{p-1}\left(2 L_{1}\right)^{\frac{p}{2}}+c_{1}\right]$. Combing (16) and (24) together, we have

$$
E \sup _{t_{0} \leq s \leq t}\left|x^{n}(s)\right|^{p} \leq c_{3}+c_{4} E \int_{t_{0}}^{t}\left\|x_{s}^{n-1}\right\|^{p} d s .
$$

where $c_{3}=\left[\frac{k_{0}}{1-k_{0}}+3^{p-1} \frac{\left(1+k_{0}\right)^{p}}{\left(1-k_{0}\right)^{p}}\right] E\|\xi\|^{p}+\frac{c_{2}}{\left(1-k_{0}\right)^{p}}\left(T-t_{0}\right)$ and $c_{4}=\frac{c_{2}}{\left(1-k_{0}\right)^{p}}$. For any $r \geq 1$,

$$
\begin{aligned}
\max _{1 \leq n \leq r} E \sup _{t_{0} \leq s \leq t}\left|x^{n}(s)\right|^{p} & \leq c_{3}+c_{4} \int_{t_{0}}^{t} \max _{1 \leq n \leq r} E \sup _{t_{0} \leq \sigma \leq s}\left|x^{n-1}(\sigma)\right|^{p} d s \\
& \leq c_{3}+c_{4} \int_{t_{0}}^{t}\left(E \|\left.\xi\right|^{p}+\max _{1 \leq n \leq r} E \sup _{t_{0} \leq \sigma \leq s}\left|x^{n}(\sigma)\right|^{p}\right) d s .
\end{aligned}
$$


From the Gronwall inequality, we derive that

$$
\max _{1 \leq n \leq r} E \sup _{t_{0} \leq t \leq T}\left|x^{n}(t)\right|^{p} \leq\left(c_{3}+c_{4}\left(T-t_{0}\right) E\|\xi\|^{p}\right) e^{c_{4}\left(T-t_{0}\right)} .
$$

Since $r$ is arbitrary, we must have

$$
E \sup _{t_{0} \leq t \leq T}\left|x^{n}(t)\right|^{p} \leq\left(c_{3}+c_{4}\left(T-t_{0}\right) E\|\xi\|^{p}\right) e^{c_{4}\left(T-t_{0}\right)},
$$

which shows that the desired result holds with $c=\left(c_{3}+c_{4}\left(T-t_{0}\right) E\|\xi\|^{p}\right) e^{c_{4}\left(T-t_{0}\right)}$.

Lemma 3.3 Let the conditions of Theorem 3.1 hold. Then $\left\{x^{n}(t)\right\}(n \geq$ 0 ) defined by (9) is a Cauchy sequence in $D\left(\left[t_{0}, T\right], R^{n}\right)$.

Proof: For $n \geq 1$ and $t \in\left[t_{0}, T\right]$, it follows from (9) that,

$$
\begin{aligned}
x^{n+1}(t)-x^{n}(t)= & D\left(x_{t}^{n+1}\right)-D\left(x_{t}^{n}\right)+\int_{t_{0}}^{t}\left[f\left(x_{s}^{n}, s\right)-f\left(x_{s}^{n-1}, s\right)\right] d s \\
& +\int_{t_{0}}^{t} \int_{U}\left[h\left(x_{s}^{n}, u\right)-h\left(x_{s}^{n-1}, u\right)\right] N_{\bar{p}}(d s, d u) .
\end{aligned}
$$

Similar to the analysis of (14), by lemma 3.1 and taking the expectation on $\left|x^{n+1}(t)-x^{n}(t)\right|^{p}$, we have

$$
\begin{aligned}
& E\left(\sup _{t_{0} \leq s \leq t}\left|x^{n+1}(s)-x^{n}(s)\right|^{p}\right) \\
\leq & k_{0} E\left(\sup _{t_{0} \leq s \leq t}\left|x^{n+1}(s)-x^{n}(s)\right|^{p}\right) \\
& +\frac{1}{\left(1-k_{0}\right)^{p-1}} E \sup _{t_{0} \leq s \leq t}\left|\left[x^{n+1}(s)-x^{n}(s)\right]-\left[D\left(x_{s}^{n+1}\right)-D\left(x_{s}^{n}\right)\right]\right|^{p} .
\end{aligned}
$$

Consequently,

$$
\begin{aligned}
& E\left(\sup _{t_{0} \leq s \leq t}\left|x^{n+1}(s)-x^{n}(s)\right|^{p}\right) \\
\leq & \frac{1}{\left(1-k_{0}\right)^{p}} E \sup _{t_{0} \leq s \leq t}\left|\left[x^{n+1}(s)-x^{n}(s)\right]-\left[D\left(x_{s}^{n+1}\right)-D\left(x_{s}^{n}\right)\right]\right|^{p} .
\end{aligned}
$$

The basic inequality $|a+b|^{p} \leq 2^{p-1}\left(|a|^{p}+|b|^{p}\right)$ implies that

$$
\begin{aligned}
& E \sup _{t_{0} \leq s \leq t}\left|\left[x^{n+1}(s)-x^{n}(s)\right]-\left[D\left(x_{s}^{n+1}\right)-D\left(x_{s}^{n}\right)\right]\right|^{p} \\
\leq & 2^{p-1}\left[E \sup _{t_{0} \leq s \leq t}\left|\int_{t_{0}}^{s}\left[f\left(x_{\sigma}^{n}, \sigma\right)-f\left(x_{\sigma}^{n-1}, \sigma\right)\right] d \sigma\right|^{p}\right. \\
& \left.+E \sup _{t_{0} \leq s \leq t}\left|\int_{t_{0}}^{s} \int_{U}\left[h\left(x_{\sigma}^{n}, u\right)-h\left(x_{\sigma}^{n-1}, u\right)\right] N_{\bar{p}}(d \sigma, d u)\right|^{p}\right] .
\end{aligned}
$$


Applying the Hölder inequality and (H2), we obtain

$$
\begin{aligned}
& E \sup _{t_{0} \leq s \leq t}\left|\int_{t_{0}}^{s}\left[f\left(x_{\sigma}^{n}, \sigma\right)-f\left(x_{\sigma}^{n-1}, \sigma\right)\right] d \sigma\right|^{p} \\
\leq & \left(t-t_{0}\right)^{p-1} E \int_{t_{0}}^{t}\left|f\left(x_{s}^{n}, s\right)-f\left(x_{s}^{n-1}, s\right)\right|^{p} d s \\
\leq & \left(t-t_{0}\right)^{p-1} k^{\frac{p}{2}} \int_{t_{0}}^{t} E \| x_{s}^{n}-x_{s}^{n-1}||^{p} d s .
\end{aligned}
$$

By the Kunita's estimates, Hölder inequality and (H2)-(H3), there exists a positive constant $c_{5}$ such that

$$
\begin{aligned}
& E \sup _{t_{0} \leq s \leq t}\left|\int_{t_{0}}^{s} \int_{U}\left[h\left(x_{\sigma}^{n}, u\right)-h\left(x_{\sigma}^{n-1}, u\right)\right] N_{\bar{p}}(d \sigma, d u)\right|^{p} \\
\leq & 2^{p-1} E \sup _{t_{0} \leq s \leq t}\left|\int_{t_{0}}^{s} \int_{U}\left[h\left(x_{\sigma}^{n}, u\right)-h\left(x_{\sigma}^{n-1}, u\right)\right] \tilde{N}_{\bar{p}}(d \sigma, d u)\right|^{p} \\
& +2^{p-1} E \sup _{t_{0} \leq s \leq t}\left|\int_{t_{0}}^{s} \int_{U}\left[h\left(x_{\sigma}^{n}, u\right)-h\left(x_{\sigma}^{n-1}, u\right)\right] \pi(d u) d \sigma\right|^{p} \\
\leq & 2^{p-1}\left[\left(t-t_{0}\right)^{p-1}(\pi(U))^{\frac{p}{2}} E \int_{t_{0}}^{t}\left[\int_{U}\left|h\left(x_{s}^{n}, u\right)-h\left(x_{s}^{n-1}, u\right)\right|^{2} \pi(d u)\right]^{\frac{p}{2}} d s\right. \\
& +c_{p} 2^{p-1}\left\{E \int_{t_{0}}^{t}\left[\int_{U}\left|h\left(x_{s}^{n}, u\right)-h\left(x_{s}^{n-1}, u\right)\right|^{2} \pi(d u)\right]^{\frac{p}{2}} d s\right. \\
& \left.+E\left[\int_{t_{0}}^{t} \int_{U}\left|h\left(x_{s}^{n}, u\right)-h\left(x_{s}^{n-1}, u\right)\right|^{p} \pi(d u) d s\right]\right\} \\
\leq & c_{5} E \int_{t_{0}}^{t}|| x_{s}^{n}-x_{s}^{n-1}||^{p} d s,
\end{aligned}
$$

where $c_{5}=2^{p-1}\left\{\left[\left(t-t_{0}\right)^{p-1}(\pi(U))^{\frac{p}{2}}+c_{p}\right] k^{\frac{p}{2}}+c_{p} L \int_{U}|u|^{p} \pi(d u)\right\}$. Hence, inserting (30)-(32) into (29) yields

$$
E\left(\sup _{t_{0} \leq s \leq t}\left|x^{n+1}(s)-x^{n}(s)\right|^{p}\right) \leq c_{6} \int_{t_{0}}^{t} E\left(\sup _{t_{0} \leq \sigma \leq s}\left|x^{n}(\sigma)-x^{n-1}(\sigma)\right|^{p}\right) d s,
$$

where $c_{6}=k^{\frac{p}{2}} 2^{p-1} \frac{1}{\left(1-k_{0}\right)^{p}}\left[\left(T-t_{0}\right)^{p-1}+c_{5}\right]$.

Setting $\varphi_{n}(t)=E \sup _{t_{0} \leq s \leq t}\left|x^{n+1}(s)-x^{n}(s)\right|^{p}$, we have

$$
\varphi_{n}(t) \leq c_{6} \int_{t_{0}}^{t} \varphi_{n-1}\left(s_{1}\right) d s_{1} \leq c_{6}^{2} \int_{0}^{t} d s_{1} \int_{t_{0}}^{s_{1}} \varphi_{n-2}\left(s_{2}\right) d s_{2}
$$




$$
\begin{aligned}
& \leq \cdots \\
& \leq c_{6}^{n} \int_{t_{0}}^{t} d s_{1} \int_{t_{0}}^{s_{1}} d s_{2} \cdots \int_{t_{0}}^{s_{n-1}} \varphi_{0}\left(s_{n}\right) d s_{n} .
\end{aligned}
$$

From the Kunita's estimates, Hölder inequality and conditions (7)-(8), we have

$$
\varphi_{0}(t)=E \sup _{t_{0} \leq s \leq t}\left|x^{1}(s)-x^{0}(s)\right|^{p} \leq c_{0} .
$$

Substituting (35) into (34) and integrating the right hand side, we obtain

$$
E\left(\sup _{t_{0} \leq s \leq t}\left|x^{n+1}(s)-x^{n}(s)\right|^{p}\right) \leq c_{0} \frac{\left(c_{6}\left(t-t_{0}\right)\right)^{n}}{n !} .
$$

Taking $t=T$ in (36), we have

$$
E\left(\sup _{t_{0} \leq t \leq T}\left|x^{n+1}(t)-x^{n}(t)\right|^{p}\right) \leq c_{0} \frac{\left(c_{6}\left(T-t_{0}\right)\right)^{n}}{n !} .
$$

Then using the Chebyshev inequality, one gets

$$
P\left(\sup _{t_{0} \leq t \leq T}\left|x^{n+1}(t)-x^{n}(t)\right|>\frac{1}{2^{n}}\right) \leq c_{0} M \frac{\left(c_{6}\left(T-t_{0}\right)\right)^{n}}{n !} .
$$

Since $\Sigma_{n=0}^{\infty} \frac{c_{0} M\left(c_{6}\left(T-t_{0}\right)\right)^{n}}{n !}<\infty$, and by the Borel-Cantelli lemma, we have

$$
P\left(\sup _{t_{0} \leq t \leq T}\left|x^{n+1}(t)-x^{n}(t)\right| \leq \frac{1}{2^{n}}\right)=1 .
$$

(38) implies that for each $t,\left\{x^{n}(t)\right\}_{n=1,2 \ldots}$ is a Cauchy sequence on $\left[t_{0}, T\right]$ under sup |.|. However, the space $D\left(\left[t_{0}, T\right], R^{n}\right)$ is not a complete space under sup $|$.$| and we cannot get the limit of the sequence \left\{x^{n}(t)\right\}_{n \geq 1}$. So we need to introduce a metric to make the space $D\left(\left[t_{0}, T\right], R^{n}\right)$ complete. For any $x, y \in D\left(\left[t_{0}, T\right], R^{n}\right)$, P.Billingsley [29] gives the following metric

$$
d(x, y)=\inf _{\lambda \in \Lambda}\left\{\sup _{t_{0} \leq t \leq T}\left|x_{t}-y_{\lambda(t)}\right|+\sup _{t_{0} \leq s \leq t \leq T}\left|\log \frac{\lambda(t)-\lambda(s)}{t-s}\right|\right\},
$$

where $\Lambda=\left\{\lambda=\lambda(t): \lambda\right.$ is strictly increasing, continuous on $t \in\left[t_{0}, T\right]$, such that $\left.\lambda\left(t_{0}\right)=t_{0}, \lambda(T)=T\right\}$. So we have that $D\left(\left[t_{0}, T\right], R^{n}\right)$ is a complete metric space. Taking $\lambda(t)=t$, we can see that $\left\{x^{n}(t)\right\}_{n \geq 1}$ is a cauchy sequence under $d(.,$.$) . The proof is completed.$ 
Proof of Theorem 3.1 Uniqueness. Let $x(t)$ and $y(t)$ be two solutions of Eq.(1). Then, for $t \in\left[t_{0}, T\right]$, by the Kunita's estimates, Hölder inequality, we have

$$
E \sup _{t_{0} \leq s \leq t}|x(s)-y(s)|^{p} \leq c \int_{t_{0}}^{t} E \sup _{t_{0} \leq u \leq s}|x(u)-y(u)|^{p} d s .
$$

Therefore, using the Gronwall inequality, we get

$$
E \sup _{t_{0} \leq s \leq t}|x(s)-y(s)|^{p}=0, \quad t \in\left[t_{0}, T\right],
$$

which implies that $x(t)=y(t)$ for all $t \in\left[t_{0}, T\right]$. Therefore, for all $t \in\left[t_{0}, T\right]$, $x(t)=y(t)$ a.s.

Existence. We derive from Lemma 3.3 that $\left\{x^{n}(t)\right\}_{n=1,2 \ldots}$ is a Cauchy sequence in $D\left(\left[t_{0}, T\right], R^{n}\right)$. Hence, there exists a unique solution $x(t) \in$ $D\left(\left[t_{0}, T\right], R^{n}\right)$ such that $d\left(x^{n}(),. x().\right) \rightarrow 0$ as $n \rightarrow \infty$. For all $t \in\left[t_{0}, T\right]$, taking limits on both sides of (9) and letting $n \rightarrow \infty$, we then can show that $x(t)$ is the solution of equation (1). So the proof of Theorem 3.1 is completed.

Next, we relax the Lipschitz conditions (H2)-(H3) and replace them by the following the local Lipschitz conditions.

(H4) For all $\varphi, \psi \in D\left([-\tau, 0] ; R^{n}\right), t \in\left[t_{0}, T\right], u \in U$ and $\|\varphi\| \vee\|\psi\| \leq n$, there exist two positive constants $k_{n}$ and $L_{0}$ such that

$$
|f(\varphi, t)-f(\psi, t)|^{2} \vee \int_{U}|h(\varphi, u)-h(\psi, u)|^{2} \pi(d u) \leq k_{n}\|\varphi-\psi\|^{2} .
$$

(H5) For all $\varphi, \psi \in D\left([-\tau, 0] ; R^{n}\right), p \geq 2, u \in U$ and $\|\varphi\| \vee\|\psi\| \leq n$, there exists a positive constant $L_{n}$ such that

$$
|h(\varphi, u)-h(\psi, u)|^{p} \leq L_{n} \| \varphi-\psi||^{p}|u|^{p} .
$$

where $\pi(U)<\infty$ and $\int_{U}|u|^{p} d u<\infty$.

Then, Theorem 3.1 can be generalized as Theorem 3.2.

Theorem 3.2 Let conditions (H1),(H4) and (H5) hold. Then equation (1) has a unique solution $x(t)$ on $\left[t_{0}, T\right]$ in the sense of $L^{p}$-norm. Moreover, there exists a constants $c$ such that

$$
E \sup _{t_{0} \leq t \leq T}|x(t)|^{p} \leq c .
$$

for any $t \in\left[t_{0}, T\right]$. 
Proof: For each $n \geq 1$, define the truncation function

$$
f_{n}(t, x)= \begin{cases}f(t, x), & \text { if } \quad\|x\| \leq n \\ f\left(t, \frac{n x}{\|x\|}\right), & \text { if }\|x\| \geq n\end{cases}
$$

and

$$
h_{n}(x, u)=\left\{\begin{array}{l}
h(x, u), \quad \text { if } \quad\|x\| \leq n, \\
h\left(\frac{n x}{\|x\|}, u\right), \quad \text { if }\|x\| \geq n .
\end{array}\right.
$$

Then $f_{n}$ and $h_{n}$ satisfy the conditions (H1)-(H3). From Theorem 3.1, we have that the following equation

$$
\begin{aligned}
x_{n}(t)= & \xi(0)+D\left(\left(x_{n}\right)_{t}\right)-D(\xi)+\int_{t_{0}}^{t} f_{n}\left(\left(x_{n}\right)_{s}, s\right) d s \\
& +\int_{t_{0}}^{t} \int_{U} h_{n}\left(\left(x_{n}\right)_{s}, u\right) N_{\bar{p}}(d s, d u)
\end{aligned}
$$

has a unique solution $x_{n}(t)$. Moreover, $x_{n+1}(t)$ is the unique solution of the equation

$$
\begin{aligned}
x_{n+1}(t)= & \xi(0)+D\left(\left(x_{n+1}\right)_{t}\right)-D(\xi)+\int_{t_{0}}^{t} f_{n+1}\left(\left(x_{n+1}\right)_{s}, s\right) d s \\
& +\int_{t_{0}}^{t} \int_{U} h_{n+1}\left(\left(x_{n+1}\right)_{s}, u\right) N_{\bar{p}}(d s, d u) .
\end{aligned}
$$

By (44) and (45), we have

$$
\begin{aligned}
& x_{n+1}(t)-x_{n}(t) \\
= & D\left(\left(x_{n+1}\right)_{t}\right)-D\left(\left(x_{n}\right)_{t}\right)+\int_{t_{0}}^{t}\left[f_{n+1}\left(\left(x_{n+1}\right)_{s}, s\right)-f_{n}\left(\left(x_{n}\right)_{s}, s\right)\right] d s \\
& +\int_{t_{0}}^{t} \int_{U}\left[h_{n+1}\left(\left(x_{n+1}\right)_{s}, u\right)-h_{n}\left(\left(x_{n}\right)_{s}, u\right)\right] N_{\bar{p}}(d s, d u) .
\end{aligned}
$$

For any fixed $n \geq 1$, define the stopping time

$$
\tau_{n}=T \wedge \inf \left\{t \in\left[t_{0}, T\right]:\left|\left(x_{n}\right)_{t}\right| \geq n\right\} .
$$


Taking the expectation on $\left|x_{n+1}(t)-x_{n}(t)\right|^{p}$ and by lemma 3.1, it deduces that

$$
\begin{aligned}
& E \sup _{t_{0} \leq s \leq t \wedge \tau_{n}}\left|x_{n+1}(s)-x_{n}(s)\right|^{p} \\
\leq & \frac{1}{\left(1-k_{0}\right)^{p-1}} E \sup _{t_{0} \leq s \leq t \wedge \tau_{n}}\left|\left[\left(x_{n+1}\right)_{s}-\left(x_{n}\right)_{s}\right]-\left[D\left(\left(x_{n+1}\right)_{s}\right)-D\left(\left(x_{n}\right)_{s}\right)\right]\right|^{p} \\
& +k_{0} E\left(\sup _{t_{0} \leq s \leq t \wedge \tau_{n}}\left|\left(x_{n+1}\right)_{s}-\left(x_{n}\right)_{s}\right|^{p}\right) .
\end{aligned}
$$

Therefore,

$$
\begin{aligned}
& E \sup _{t_{0} \leq s \leq t \wedge \tau_{n}}\left|x_{n+1}(s)-x_{n}(s)\right|^{p} \\
\leq & \frac{1}{\left(1-k_{0}\right)^{p}} 2^{p-1}\left\{E \left(\sup _{t_{0} \leq s \leq t \wedge \tau_{n}}\left|\int_{t_{0}}^{s}\left[f_{n+1}\left(\left(x_{n+1}\right)_{\sigma}, \sigma\right)-f_{n}\left(\left(x_{n}\right)_{\sigma}, \sigma\right)\right] d \sigma\right|^{p}\right.\right. \\
& +E\left(\sup _{t_{0} \leq s \leq t \wedge \tau_{n}}\left|\int_{t_{0}}^{s} \int_{U}\left[h_{n+1}\left(\left(x_{n+1}\right)_{\sigma}, u\right)-h_{n}\left(\left(x_{n}\right)_{\sigma}, u\right)\right] N_{\bar{p}}(d \sigma, d u)\right|^{p}\right\} \\
= & \frac{1}{\left(1-k_{0}\right)^{p}} 2^{p-1}\left(J_{1}+J_{2}\right) .
\end{aligned}
$$

By the Hölder inequality and rearranging the terms on the right-hand side by plus and minus technique, we have

$$
\begin{aligned}
J_{1} \leq & \left(t \wedge \tau_{n}-t_{0}\right)^{p-1} E \int_{t_{0}}^{t \wedge \tau_{n}}\left|f_{n+1}\left(\left(x_{n+1}\right)_{s}, s\right)-f_{n}\left(\left(x_{n}\right)_{s}, s\right)\right|^{p} d s \\
\leq & \left(t \wedge \tau_{n}-t_{0}\right)^{p-1} E \int_{t_{0}}^{t \wedge \tau_{n}}\left\{2^{p-1}\left|f_{n+1}\left(\left(x_{n+1}\right)_{s}, s\right)-f_{n+1}\left(\left(x_{n}\right)_{s}, s\right)\right|^{p}\right. \\
& \left.+2^{p-1}\left|f_{n+1}\left(\left(x_{n}\right)_{s}, s\right)-f_{n}\left(\left(x_{n}\right)_{s}, s\right)\right|^{p}\right\} d s
\end{aligned}
$$

The Kunita's estimates implies that

$$
\begin{aligned}
J_{2} \leq & c_{7} E \int_{t_{0}}^{t \wedge \tau_{n}}\left[\int_{U}\left|h_{n+1}\left(\left(x_{n+1}\right)_{s}, u\right)-h_{n}\left(\left(x_{n}\right)_{s}, u\right)\right|^{2} \pi(d u)\right]^{\frac{p}{2}} d s \\
& +c_{p} 2^{p-1} E \int_{t_{0}}^{t \wedge \tau_{n}} \int_{U}\left|h_{n+1}\left(\left(x_{n+1}\right)_{s}, u\right)-h_{n}\left(\left(x_{n}\right)_{s}, u\right)\right|^{p} \pi(d u) d s \\
\leq & c_{7} E \int_{t_{0}}^{t \wedge \tau_{n}}\left\{\int _ { U } \left[2\left|h_{n+1}\left(\left(x_{n+1}\right)_{s}, u\right)-h_{n+1}\left(\left(x_{n}\right)_{s}, u\right)\right|^{2}\right.\right.
\end{aligned}
$$




$$
\begin{aligned}
& \left.\left.+2\left|h_{n+1}\left(\left(x_{n}\right)_{s}, u\right)-h_{n}\left(\left(x_{n}\right)_{s}, u\right)\right|^{2}\right] \pi(d u)\right\}^{\frac{p}{2}} d s \\
& +c_{p} 2^{2 p-2}\left\{E \int_{t_{0}}^{t \wedge \tau_{n}} \int_{U}\left|h_{n+1}\left(\left(x_{n+1}\right)_{s}, u\right)-h_{n+1}\left(\left(x_{n+1}\right)_{s}, u\right)\right|^{p} \pi(d u) d s\right. \\
& \left.+E \int_{t_{0}}^{t \wedge \tau_{n}} \int_{U}\left|h_{n+1}\left(\left(x_{n+1}\right)_{s}, u\right)-h_{n}\left(\left(x_{n}\right)_{s}, u\right)\right|^{p} \pi(d u) d s\right\}
\end{aligned}
$$

where $c_{7}=2^{p-1}\left[\left(t \wedge \tau_{n}-t_{0}\right)^{p-1}(\pi(U))^{\frac{p}{2}}+c_{p}\right]$. Combing (48)-(50) together, it follows that

$$
\begin{aligned}
& E \sup _{t_{0} \leq s \leq t \wedge \tau_{n}}\left|x_{n+1}(s)-x_{n}(s)\right|^{p} \\
\leq & \frac{1}{\left(1-k_{0}\right)^{p}} 2^{p-1}\left(T-t_{0}\right)^{p-1} E \int_{t_{0}}^{t \wedge \tau_{n}}\left\{2^{p-1} \mid f_{n+1}\left(\left(x_{n+1}\right)_{s}, s\right)\right. \\
& \left.-\left.f_{n+1}\left(\left(x_{n}\right)_{s}, s\right)\right|^{p}+2^{p-1}\left|f_{n+1}\left(\left(x_{n}\right)_{s}, s\right)-f_{n}\left(\left(x_{n}\right)_{s}, s\right)\right|^{p}\right\} d s \\
& +\frac{1}{\left(1-k_{0}\right)^{p}} 2^{p-1}\left\{c _ { 7 } E \int _ { t _ { 0 } } ^ { t \wedge \tau _ { n } } \left\{\int _ { U } \left[2\left|h_{n+1}\left(\left(x_{n+1}\right)_{s}, u\right)-h_{n+1}\left(\left(x_{n}\right)_{s}, u\right)\right|^{2}\right.\right.\right. \\
& \left.\left.+2\left|h_{n+1}\left(\left(x_{n}\right)_{s}, u\right)-h_{n}\left(\left(x_{n}\right)_{s}, u\right)\right|^{2}\right] \pi(d u)\right\}^{\frac{p}{2}} d s \\
& +c_{p} 2^{2 p-2}\left\{E \int_{t_{0}}^{t \wedge \tau_{n}} \int_{U}\left|h_{n+1}\left(\left(x_{n+1}\right)_{s}, u\right)-h_{n+1}\left(\left(x_{n+1}\right)_{s}, u\right)\right|^{p} \pi(d u) d s\right. \\
& \left.\left.+E \int_{t_{0}}^{t \wedge \tau_{n}} \int_{U}\left|h_{n+1}\left(\left(x_{n+1}\right)_{s}, u\right)-h_{n}\left(\left(x_{n}\right)_{s}, u\right)\right|^{p} \pi(d u) d s\right\}\right\} .
\end{aligned}
$$

For $t_{0} \leq t \leq \tau_{n}$, we have

$$
\begin{aligned}
f_{n+1}\left(\left(x_{n}\right)_{t}, t\right)=f_{n}\left(\left(x_{n}\right)_{t}, t\right) & =f\left(\left(x_{n}\right)_{t}, t\right), \\
h_{n+1}\left(\left(x_{n}\right)_{t}, u\right)=h_{n}\left(\left(x_{n}\right)_{t}, u\right) & =h\left(\left(x_{n}\right)_{t}, u\right) .
\end{aligned}
$$

By (52), we get from (51),

$$
\begin{aligned}
& E \sup _{t_{0} \leq s \leq t \wedge \tau_{n}}\left|x_{n+1}(s)-x_{n}(s)\right|^{p} \\
\leq & \frac{1}{\left(1-k_{0}\right)^{p}} 2^{2 p-2}\left(T-t_{0}\right)^{p-1} E \int_{t_{0}}^{t \wedge \tau_{n}}\left|f_{n+1}\left(\left(x_{n+1}\right)_{s}, s\right)-f_{n+1}\left(\left(x_{n}\right)_{s}, s\right)\right|^{p} d s \\
& +\frac{1}{\left(1-k_{0}\right)^{p}} 2^{p-1}\left\{c _ { 7 } E \int _ { t _ { 0 } } ^ { t \wedge \tau _ { n } } \left\{\int _ { U } \left[2 \mid h_{n+1}\left(\left(x_{n+1}\right)_{s}, u\right)\right.\right.\right. \\
& \left.\left.-\left.h_{n+1}\left(\left(x_{n}\right)_{s}, u\right)\right|^{2}\right] \pi(d u)\right\}^{\frac{p}{2}} d s
\end{aligned}
$$




$$
\left.+c_{p} 2^{2 p-2} E \int_{t_{0}}^{t \wedge \tau_{n}} \int_{U}\left|h_{n+1}\left(\left(x_{n+1}\right)_{s}, u\right)-h_{n+1}\left(\left(x_{n+1}\right)_{s}, u\right)\right|^{p} \pi(d u) d s\right\} .
$$

By the local Lipschitz conditions (H4) and (H5), we have

$$
\begin{aligned}
E \sup _{t_{0} \leq s \leq t \wedge \tau_{n}}\left|x_{n+1}(s)-x_{n}(s)\right|^{p} & \leq c_{8} E \int_{t_{0}}^{t \wedge \tau_{n}} \|\left(x_{n+1}\right)_{s}-\left.\left(x_{n}\right)_{s}\right|^{p} d s \\
& \leq c_{8} \int_{t_{0}}^{t} E \sup _{t_{0} \leq \sigma \leq s \wedge \tau_{n}}\left|x_{n+1}(\sigma)-x_{n}(\sigma)\right|^{p} d s,
\end{aligned}
$$

where $c_{8}=\frac{1}{\left(1-k_{0}\right)^{p}}\left\{k_{n+1}^{\frac{p}{2}}\left[2^{2 p-2}\left(T-t_{0}\right)^{p-1}+2^{\frac{3}{2} p-2} c_{7}\right]+c_{p} 2^{3 p-3} L_{n+1} \int_{U}|u|^{p} \pi(d u)\right\}$. From (54) and the Gronwall inequality, we get

$$
E \sup _{t_{0} \leq s \leq t \wedge \tau_{n}}\left|x_{n+1}(s)-x_{n}(s)\right|^{p}=0
$$

which yields

$$
x_{n+1}(t)=x_{n}(t), \quad \text { for } t \in\left[t_{0}, \tau_{n}\right] .
$$

It then deduced that $\tau_{n}$ is increasing, that is as $n \rightarrow \infty, \tau_{n} \uparrow T$ a.s. By the linear growth condition (7) and (8), for almost all $\omega \in \Omega$, there exists an integer $n_{0}=n_{0}(\omega)$ such that $\tau_{n}=T$ as $n \geq n_{0}$. Now define $x(t)$ by $x(t)=x_{n_{0}}(t)$ for $t \in\left[t_{0}, T\right]$. Next to verify that $x(t)$ is the solution of equation (1). By (56), $x\left(t \wedge \tau_{n}\right)=x_{n}\left(t \wedge \tau_{n}\right)$, and it follows from (44) that

$$
\begin{aligned}
& x\left(t \wedge \tau_{n}\right)-D\left(x_{t \wedge \tau_{n}}\right) \\
= & \xi(0)-D(\xi)+\int_{t_{0}}^{t \wedge \tau_{n}} f_{n}\left(x_{s}, s\right) d s+\int_{t_{0}}^{t \wedge \tau_{n}} \int_{U} h_{n}\left(x_{s}, u\right) N_{\bar{p}}(d s, d u) \\
= & \xi(0)-D(\xi)+\int_{t_{0}}^{t \wedge \tau_{n}} f\left(x_{s}, s\right) d s+\int_{t_{0}}^{t \wedge \tau_{n}} \int_{U} h\left(x_{s}, u\right) N_{\bar{p}}(d s, d u) .
\end{aligned}
$$

Letting $n \rightarrow \infty$ on both sides of (57), we obtain

$$
\begin{aligned}
& x(t \wedge T)-D\left(x_{t \wedge T}\right) \\
= & \xi(0)-D(\xi)+\int_{t_{0}}^{t \wedge T} f\left(x_{s}, s\right) d s+\int_{t_{0}}^{t \wedge T} \int_{U} h\left(x_{s}, u\right) N_{\bar{p}}(d s, d u) .
\end{aligned}
$$


that is

$$
x(t)-D\left(x_{t}\right)=\xi(0)-D(\xi)+\int_{t_{0}}^{t} f\left(x_{s}, s\right) d s+\int_{t_{0}}^{t} \int_{U} h\left(x_{s}, u\right) N_{\bar{p}}(d s, d u),
$$

which implies that $x(t)$ is the solution of equation (1). By stopping our process $x(t)$, uniqueness of the solution to equation (1) is obtained. Moreover, by the proof of Theorem 3.1, we can easily obtain that $E \sup _{t_{0} \leq t \leq T}|x(t)|^{p} \leq c$.

The proof is completed.

\section{Asymptotic estimations for solutions}

In this section, we will give the exponential estimate of the solution to equation (1).

According to the definition of $\tilde{N}_{\bar{p}}(d t, d u):=N_{\bar{p}}(d t, d u)-\pi(d u) d t$, we can rewrite equation (1) as the following equation

$$
d\left[x(t)-D\left(x_{t}\right)\right]=F\left(t, x_{t}\right) d t+\int_{U} h\left(x_{t}, u\right) \tilde{N}_{\bar{p}}(d t, d u),
$$

where $F\left(t, x_{t}\right)=f\left(x_{t}, t\right)+\int_{U} h\left(x_{t}, u\right) \pi(d u)$.

Let $C^{2,1}\left(R^{n} \times\left[t_{0}-\tau, T\right), R_{+}\right)$denote the family of all nonnegative functions $V(x, t)$ on $R^{n} \times\left[t_{0}-\tau, T\right)$ which are continuously twice differentiable with respect to $x$ and continuously once differentiable with respect to $t$. For a $V \in C^{2,1}\left(R^{n} \times\left[t_{0}-\tau, T\right), R_{+}\right)$, one can define the Kolmogorov operator $L V$ as follows:

$$
\begin{aligned}
L V(x, y, t) \equiv & V_{t}(x-D(y), t)+V_{x}(x-D(y), t) F(t, y) \\
& +\int_{U}[V(x-D(y)+h(y, u), t)-V(x-D(y), t) \\
& \left.-V_{x}(x-D(y), t) h(y, u)\right] \pi(d u),
\end{aligned}
$$

where

$$
V_{t}(x, t)=\frac{\partial V(x, t)}{\partial t}, \quad V_{x}(x, t)=\left(\frac{\partial V(x, t)}{\partial x_{1}}, \cdots, \frac{\partial V(x, t)}{\partial x_{n}}\right)
$$

First, we establish the $p$-th exponential estimations of the solution to equation (1). 
Theorem 4.1 Let $\left\{x(t), t_{0} \leq t \leq T\right\}$ be a solution of equation (1) whose coefficients satisfy conditions (H1) and (H2). For a given integer $p \geq 2$ and any $2 \leq q \leq p$, there exists a positive constant $K$ such that

$$
\int_{U}|h(\varphi, u)|^{q} \pi(d u) \leq K\|\varphi\|^{q} .
$$

Then, for any $t_{0} \leq t \leq T$,

$$
E \sup _{t_{0}-\tau \leq s \leq t}|x(s)|^{p} \leq\left[1+\left(1+c_{12}\right) E \| \xi||^{p}\right] e^{M\left(t-t_{0}\right)},
$$

where $M=\frac{2\left(c_{9}+c_{10}+c_{11}\right)}{\left(1-k_{0}\right)^{p}} . c_{9}, c_{10}, c_{11}, c_{12}$ are four positive constants of (68), (74), (80), (82).

Proof: Let $V\left(x(t)-D\left(x_{t}\right), t\right)=1+\left|x(t)-D\left(x_{t}\right)\right|^{p}$, then $V_{t}(x(t)-$ $\left.D\left(x_{t}\right), t\right)=0$. Applying the It $\widehat{o}$ formula to $V\left(x(t)-D\left(x_{t}\right), t\right)$, we obtain that

$$
\begin{aligned}
V\left(x(t)-D\left(x_{t}\right), t\right)= & V\left(x\left(t_{0}-D\left(x_{t_{0}}\right)\right), t_{0}\right)+\int_{t_{0}}^{t} L V\left(x(s), x_{s}, s\right) d s \\
& +\int_{t_{0}}^{t} \int_{U}\left[V\left(x(s)-D\left(x_{s}\right)+h\left(x_{s}, u\right), s\right)\right. \\
& \left.-V\left(x(s)-D\left(x_{s}\right), s\right)\right] \tilde{N}_{\bar{p}}(d s, d u) .
\end{aligned}
$$

By (59), we have

$$
\begin{aligned}
& 1+\left|x(t)-D\left(x_{t}\right)\right|^{p}=1+\left|x\left(t_{0}\right)-D\left(x_{t_{0}}\right)\right|^{p} \\
& +p \int_{t_{0}}^{t}\left|x(s)-D\left(x_{s}\right)\right|^{p-2}\left[x(s)-D\left(x_{s}\right)\right]^{\top} F\left(s, x_{s}\right) d s \\
& +\int_{t_{0}}^{t} \int_{U}\left\{\left(1+\left|x(s)-D\left(x_{s}\right)+h\left(x_{s}, u\right)\right|^{p}\right)-\left(1+\left|x(s)-D\left(x_{s}\right)\right|^{p}\right)\right. \\
& \left.-p\left|x(s)-D\left(x_{s}\right)\right|^{p-2}\left[x(s)-D\left(x_{s}\right)\right]^{\top} h\left(x_{s}, u\right)\right\} \pi(d u) d s \\
& +\int_{t_{0}}^{t} \int_{U}\left\{\left(1+\left|x(s)-D\left(x_{s}\right)+h\left(x_{s}, u\right)\right|^{p}\right)\right. \\
& \left.-\left(1+\left|x(s)-D\left(x_{s}\right)\right|^{p}\right)\right\} \tilde{N}_{\bar{p}}(d s, d u) .
\end{aligned}
$$

Taking the expectation on both sides of (63), one gets

$$
E \sup _{t_{0} \leq s \leq t}\left(1+\left|x(s)-D\left(x_{s}\right)\right|^{p}\right)
$$




$$
\begin{aligned}
\leq & 1+E \sup _{t_{0} \leq s \leq t}|\xi+D(\xi)|^{p}+p E \int_{t_{0}}^{t}\left|x(s)-D\left(x_{s}\right)\right|^{p-1}\left|F\left(s, x_{s}\right)\right| d s \\
& +p E \sup _{t_{0} \leq s \leq t} \int_{t_{0}}^{s} \int_{U}\left|x(\sigma)-D\left(x_{\sigma}\right)\right|^{p-2}\left[x(\sigma)-D\left(x_{\sigma}\right)\right]^{\top} h\left(x_{\sigma}, u\right) \tilde{N}_{\bar{p}}(d \sigma, d u) \\
& +E \sup _{t_{0} \leq s \leq t} \int_{t_{0}}^{s} \int_{U}\left\{\left|x(\sigma)-D\left(x_{\sigma}\right)+h\left(x_{\sigma}, u\right)\right|^{p}-\left|x(\sigma)-D\left(x_{\sigma}\right)\right|^{p}\right. \\
& \left.-p\left|x(\sigma)-D\left(x_{\sigma}\right)\right|^{p-2}\left[x(\sigma)-D\left(x_{\sigma}\right)\right]^{\top} h\left(x_{\sigma}, u\right)\right\} N_{\bar{p}}(d \sigma, d u) \\
\leq & 1+\left(1+k_{0}\right)^{p} E \| \xi||^{p}+\sum_{i=1}^{3} Q_{i},
\end{aligned}
$$

where

$$
\begin{aligned}
Q_{1}= & p E \int_{t_{0}}^{t}\left|x(s)-D\left(x_{s}\right)\right|^{p-1}\left|F\left(s, x_{s}\right)\right| d s, \\
Q_{2}= & p E \sup _{t_{0} \leq s \leq t} \int_{t_{0}}^{s} \int_{U}\left|x(\sigma)-D\left(x_{\sigma}\right)\right|^{p-2}\left[x(\sigma)-D\left(x_{\sigma}\right)\right]^{\top} h\left(x_{\sigma}, u\right) \tilde{N}_{\bar{p}}(d \sigma, d u), \\
Q_{3}= & E \sup _{t_{0} \leq s \leq t} \int_{t_{0}}^{s} \int_{U}\left\{\left|x(\sigma)-D\left(x_{\sigma}\right)+h\left(x_{\sigma}, u\right)\right|^{p}-\left|x(\sigma)-D\left(x_{\sigma}\right)\right|^{p}\right. \\
& \left.-p\left|x(\sigma)-D\left(x_{\sigma}\right)\right|^{p-2}\left[x(\sigma)-D\left(x_{\sigma}\right)\right]^{\top} h\left(x_{\sigma}, u\right)\right\} N_{\bar{p}}(d \sigma, d u) .
\end{aligned}
$$

Let us estimate $Q_{1}$. By the basic inequality

$$
a^{r} b^{1-r} \leq r a+(1-r) b, \quad r \in[0,1],
$$

we derive that

$$
a^{p-1} b \leq \frac{\varepsilon_{1}(p-1)}{p} a^{p}+\frac{1}{p \varepsilon_{1}^{p-1}} b^{p},
$$

where $a, b, \varepsilon_{1}>0$. Hence,

$$
\begin{aligned}
Q_{1} & \leq p E \int_{t_{0}}^{t}\left[\frac{\varepsilon_{1}(p-1)}{p}\left|x(s)-D\left(x_{s}\right)\right|^{p}+\frac{1}{p \varepsilon_{1}^{p-1}}\left|F\left(s, x_{s}\right)\right|^{p}\right] d s \\
& \leq p E \int_{t_{0}}^{t}\left[\left.\frac{\varepsilon_{1}(p-1)}{p}\left(1+k_{0}\right)^{p}|| x_{s}\right|^{p}+\frac{1}{p \varepsilon_{1}^{p-1}}\left|F\left(s, x_{s}\right)\right|^{p}\right] d s .
\end{aligned}
$$

By using lemma 3.1, we have

$$
E \int_{t_{0}}^{t}\left|F\left(s, x_{s}\right)\right|^{p} d s
$$




$$
\begin{aligned}
& \leq E \int_{t_{0}}^{t}\left[1+\varepsilon^{\frac{1}{p-1}}\right]^{p-1}\left[\left|\int_{U} h\left(x_{s}, u\right) \pi(d u)\right|^{p}+\frac{\left|f\left(x_{s}, s\right)\right|^{p}}{\varepsilon}\right] d s . \\
& \leq(2 L)^{\frac{p}{2}} E \int_{t_{0}}^{t}\left[1+\varepsilon^{\frac{1}{p-1}}\right]^{p-1}\left[\left((\pi(U))^{\frac{p}{2}}+\frac{1}{\varepsilon}\right)\left(1+\left\|x_{s}\right\|^{p}\right)\right] d s .
\end{aligned}
$$

Letting $\varepsilon=(2 L)^{p-1}$, then we get

$$
E \int_{t_{0}}^{t}\left|F\left(s, x_{s}\right)\right|^{p} d s \leq(1+2 L)^{\frac{3 p}{2}-1}(\pi(U))^{\frac{p}{2}} E \int_{t_{0}}^{t}\left(1+\left\|x_{s}\right\|^{p}\right) d s .
$$

Inserting (67) into (65) and letting $\varepsilon_{1}=\frac{1+2 L}{1+k_{0}}$, we obtain that

$$
\begin{aligned}
Q_{1} \leq & p E \int_{t_{0}}^{t}\left[\frac{\varepsilon_{1}(p-1)\left(1+k_{0}\right)^{p}}{p}\left\|x_{s}\right\|^{p}\right. \\
& \left.+\frac{(1+2 L)^{\frac{3 p}{2}-1}(\pi(U))^{\frac{p}{2}}}{p \varepsilon_{1}^{\frac{p-2}{2}}}\left(1+\left\|x_{s}\right\|^{p}\right)\right] d s \\
\leq & c_{9} E \int_{t_{0}}^{t}\left(1+\left\|x_{s}\right\|^{p}\right) d s,
\end{aligned}
$$

where $c_{9}=(1+2 L)^{p}\left(1+k_{0}\right)^{p-1}\left[p+(\pi(U))^{\frac{p}{2}}\right]$. For the estimation of $Q_{2}$. By using the Burkholder-Davis inequality, there exists a positive constant $\tilde{c}_{p}$ such that

$$
\begin{aligned}
Q_{2} \leq & p \tilde{c}_{p} E\left[\int_{t_{0}}^{t} \int_{U}\left|x(s)-D\left(x_{s}\right)\right|^{2 p-2}\left|h\left(x_{s}, u\right)\right|^{2} \pi(d u) d s\right]^{\frac{1}{2}} \\
\leq & p \tilde{c}_{p} E\left[\operatorname { s u p } _ { t _ { 0 } \leq s \leq t } | x ( s ) - D ( x _ { s } ) | ^ { p } \left(\int_{t_{0}}^{t} \int_{U}\left|x(s)-D\left(x_{s}\right)\right|^{p-2}\right.\right. \\
& \left.\left.\times\left|h\left(x_{s}, u\right)\right|^{2} \pi(d u) d s\right)\right]^{\frac{1}{2}} .
\end{aligned}
$$

Further, for any $\varepsilon>0$, the Young inequality implies that

$$
\begin{aligned}
Q_{2} \leq & p \tilde{c}_{p}\left[\varepsilon E \sup _{t_{0} \leq s \leq t}\left|x(s)-D\left(x_{s}\right)\right|^{p}\right]^{\frac{1}{2}}\left[\frac { 1 } { \varepsilon } E \left(\int_{t_{0}}^{t} \int_{U}\left|x(s)-D\left(x_{s}\right)\right|^{p-2}\right.\right. \\
& \left.\left.\times\left|h\left(x_{s}, u\right)\right|^{2} \pi(d u) d s\right)\right]^{\frac{1}{2}} \\
\leq & \frac{p \tilde{c}_{p} \varepsilon}{2} E \sup _{t_{0} \leq s \leq t}\left|x(s)-D\left(x_{s}\right)\right|^{p}+\frac{p \tilde{c}_{p}}{2 \varepsilon} E \int_{t_{0}}^{t} \int_{U}\left|x(s)-D\left(x_{s}\right)\right|^{p-2} \\
& \times\left|h\left(x_{s}, u\right)\right|^{2} \pi(d u) d s .
\end{aligned}
$$


Letting $\varepsilon=\frac{1}{p \tilde{c}_{p}}$, we obtain

$$
\begin{aligned}
Q_{2} \leq & \frac{1}{2} E \sup _{t_{0} \leq s \leq t}\left|x(s)-D\left(x_{s}\right)\right|^{p}+\frac{1}{2} p^{2} \tilde{c}_{p}^{2} E \int_{t_{0}}^{t} \int_{U}\left|x(s)-D\left(x_{s}\right)\right|^{p-2} \\
& \times\left|h\left(x_{s}, u\right)\right|^{2} \pi(d u) d s .
\end{aligned}
$$

By the following inequality (see Mao[2]),

$$
a^{p-2} b^{2} \leq \frac{\varepsilon_{2}(p-2)}{p} a^{p}+\frac{1}{p \varepsilon_{2}^{\frac{p-2}{2}}} b^{p}, \quad a, b, \varepsilon_{2}>0,
$$

and condition (60), we have

$$
\begin{aligned}
& E \int_{t_{0}}^{t} \int_{U}\left|x(s)-D\left(x_{s}\right)\right|^{p-2}\left|h\left(x_{s}, u\right)\right|^{2} \pi(d u) d s \\
\leq & \frac{(p-2) \varepsilon_{2}}{p} E \int_{t_{0}}^{t} \int_{U}\left|x(s)-D\left(x_{s}\right)\right|^{p} \pi(d u) d s \\
& \left.+\frac{2}{p \varepsilon_{2}^{\frac{p-2}{2}}} E \int_{0}^{t} \int_{U}\left|h\left(x_{s}, u\right)\right|^{p} \pi(d u) d s\right] \\
\leq & {\left[\frac{(p-2) \varepsilon_{2}\left(1+k_{0}\right)^{p}}{p}\right] E \int_{t_{0}}^{t} \int_{U}\left\|x_{s}\right\|^{p} \pi(d u) d s } \\
& \left.+\frac{2}{p \varepsilon_{2}^{\frac{p-2}{2}}} K E \int_{0}^{t} \|\left. x_{s}\right|^{p} d s\right]
\end{aligned}
$$

Letting $\varepsilon_{2}=\frac{1}{\left(1+k_{0}\right)^{2}}$,

$$
\begin{aligned}
& E \int_{t_{0}}^{t} \int_{U}\left|x(s)-D\left(x_{s}\right)\right|^{p-2}\left|h\left(x_{s}, u\right)\right|^{2} \pi(d u) d s \\
\leq & {\left[\frac{(p-2)}{p} \pi(U)+\frac{2 K}{p}\right]\left(1+k_{0}\right)^{p-2} E \int_{t_{0}}^{t}\left\|x_{s}\right\|^{p} d s . }
\end{aligned}
$$

Inserting (73) into (71), we obtain

$$
Q_{2} \leq c_{10} E \int_{t_{0}}^{t}\left\|x_{s}\right\|^{p} d s+\frac{1}{2} E \sup _{t_{0} \leq s \leq t}\left|x(s)-D\left(x_{s}\right)\right|^{p},
$$


where $c_{10}=\frac{1}{2} p \tilde{c}_{p}^{2}[(p-2) \pi(U)+2 K]\left(1+k_{0}\right)^{p-2}$. Finally, let us estimate $Q_{3}$. Since $N_{\bar{p}}(d t, d u)=\tilde{N}_{\bar{p}}(d t, d u)+\pi(d u) d t$ and $\tilde{N}_{\bar{p}}(d t, d u)$ is a martingale measure, we get

$$
\begin{aligned}
Q_{3}= & E \int_{t_{0}}^{t} \int_{U}\left\{\left|x(s)-D\left(x_{s}\right)+h\left(x_{s}, u\right)\right|^{p}-\left|x(s)-D\left(x_{s}\right)\right|^{p}\right. \\
& -p\left|x(s)-D\left(x_{s}\right)\right|^{p-2}\left[x(s)-D\left(x_{s}\right]^{\top} h\left(x_{s}, u\right)\right\} \pi(d u) d s .
\end{aligned}
$$

We note that it has the form

$$
\begin{array}{r}
E \int_{t_{0}}^{t} \int_{U}\left\{f\left(x(s)-D\left(x_{s}\right)+h\left(x_{s}, u\right)\right)-f\left(x(s)-D\left(x_{s}\right)\right)\right. \\
\left.-f^{\prime}\left(x(s)-D\left(x_{s}\right)\right) h\left(x_{s}, u\right)\right\} \pi(d u) d s,
\end{array}
$$

where $f(x)=|x|^{p}$. Using the Taylor formula, there exists a positive constant $M_{p}$, such that for $p \geq 2$

$$
\begin{aligned}
& f\left(x(s)-D\left(x_{s}\right)+h\left(x_{s}, u\right)\right)-f\left(x(s)-D\left(x_{s}\right)\right) \\
& -f^{\prime}\left(x(s)-D\left(x_{s}\right)\right) h\left(x_{s}, u\right) \\
= & \left|x(s)-D\left(x_{s}\right)+h\left(x_{s}, u\right)\right|^{p}-\left|x(s)-D\left(x_{s}\right)\right|^{p} \\
& -p\left|x(s)-D\left(x_{s}\right)\right|^{p-2}\left[x(s)-D\left(x_{s}\right)\right]^{\top} h\left(x_{s}, u\right) \\
\leq & M_{p}\left[\left|x(s)-D\left(x_{s}\right)+h\left(x_{s}, u\right)\right|^{p-2}\left|h\left(x_{s}, u\right)\right|^{2}\right] .
\end{aligned}
$$

Again the basic inequality $|a+b|^{p-2} \leq 2^{p-3}\left(|a|^{p-2}+|b|^{p-2}\right)$ and the Young inequality implies that

$$
\begin{aligned}
Q_{3} \leq & M_{p} E \int_{t_{0}}^{t} \int_{U}\left[\left|x(s)-D\left(x_{s}\right)+h\left(x_{s}, u\right)\right|^{p-2}\left|h\left(x_{s}, u\right)\right|^{2}\right] \pi(d u) d s \\
\leq & M_{p} 2^{p-3} E \int_{t_{0}}^{t} \int_{U}\left[\left(\left|x(s)-D\left(x_{s}\right)\right|^{p-2}+\left|h\left(x_{s}, u\right)\right|^{p-2}\right)\left|h\left(x_{s}, u\right)\right|^{2}\right] \pi(d u) d s \\
\leq & M_{p} 2^{p-3} \frac{p-2}{p}\left(1+k_{0}\right)^{p-2} \pi(U) E \int_{t_{0}}^{t} \| x_{s}||^{p} d s \\
& +M_{p} 2^{p-3}\left(1+\frac{2\left(1+k_{0}\right)^{p-2}}{p}\right) E \int_{t_{0}}^{t} \int_{U}\left|h\left(x_{s}, u\right)\right|^{p} \pi(d u) d s
\end{aligned}
$$

By (60), we have

$$
E \int_{t_{0}}^{t} \int_{U}\left|h\left(x_{s}, u\right)\right|^{p} \pi(d u) d s \leq K E \int_{t_{0}}^{t}\left\|x_{s}\right\|^{p} d s .
$$


Substituting (79) into (78),

$$
Q_{3} \leq c_{11} \int_{t_{0}}^{t} E\left\|x_{s}\right\|^{p} d s
$$

where $c_{11}=M_{p} 2^{p-3}\left[\frac{p-2}{p}\left(1+k_{0}\right)^{p-2} \pi(U)+\left(1+\frac{2\left(1+k_{0}\right)^{p-2}}{p}\right) K\right]$. Combing (64), (68), (74) and (80) together, we obtain that

$$
\begin{aligned}
E \sup _{t_{0} \leq s \leq t}\left(1+\left|x(s)-D\left(x_{s}\right)\right|^{p}\right) \leq & 2+2\left(1+k_{0}\right)^{p} E\|\xi\|^{p} \\
& +2\left(c_{9}+c_{10}+c_{11}\right) \int_{t_{0}}^{t} E\left(1+\left\|x_{s}\right\|^{p}\right) d s .
\end{aligned}
$$

On the other hand, by lemma 3.1, we have

$$
\begin{aligned}
E \sup _{t_{0} \leq s \leq t}|x(s)|^{p} \leq & \frac{k_{0}}{1-k_{0}} E\|\xi\|^{p} \\
& +\frac{1}{\left(1-k_{0}\right)^{p}} E \sup _{t_{0} \leq s \leq t}\left(1+\left|x(s)-D\left(x_{s}\right)\right|^{p}\right) \\
\leq & c_{12} E\|\xi\|^{p}+\frac{2\left(c_{9}+c_{10}+c_{11}\right)}{\left(1-k_{0}\right)^{p}} \int_{t_{0}}^{t} E\left(1+\left\|x_{s}\right\|^{p}\right) d s,
\end{aligned}
$$

where $c_{12}=\frac{k_{0}}{1-k_{0}}+\frac{2+2\left(1+k_{0}\right)^{p}}{\left(1-k_{0}\right)^{p}}$. Consequently,

$$
\begin{aligned}
E\left(1+\sup _{t_{0}-\tau \leq s \leq t}|x(s)|^{p}\right) \leq & 1+\left(1+c_{12}\right) E\|\xi\|^{p} \\
& +\frac{2\left(c_{9}+c_{10}+c_{11}\right)}{\left(1-k_{0}\right)^{p}} \int_{t_{0}}^{t} E\left(1+\sup _{t_{0}-\tau \leq \sigma \leq s}|x(\sigma)|^{p}\right) d s .
\end{aligned}
$$

Therefore, we apply the Gronwall inequality to get

$$
E\left(1+\sup _{t_{0}-\tau \leq s \leq t}|x(s)|^{p}\right) \leq\left[1+\left(1+c_{12}\right) E \| \xi||^{p}\right] e^{M\left(t-t_{0}\right)},
$$

where $M=\frac{2\left(c_{9}+c_{10}+c_{11}\right)}{\left(1-k_{0}\right)^{p}}$. This completes the proof.

The next result shows that exponential estimations implies almost surely asymptotic estimations, and we give an upper bound for the sample Lyapunov exponent. 
Theorem 4.2 Under the conditions (H1)-(H2), we have

$$
\limsup _{t \rightarrow \infty} \frac{1}{t} \log |x(t)| \leq \frac{L\left[\left(1+k_{0}^{2}\right)+3+2 \pi(U)+2 \tilde{c}_{2}^{2}\right]}{\left(1-k_{0}\right)^{2}}, \quad \text { a.s. }
$$

That is, the sample Lyapunov exponent of the solution should not be greater than $\frac{L\left[\left(1+k_{0}^{2}\right)+3+2 \pi(U)+2 \tilde{c}_{2}^{2}\right]}{\left(1-k_{0}\right)^{2}}$.

Proof: For each $n=1,2, \ldots$, it follows from Theorem 4.1 (taking $p=2$ ) that

$$
E\left(\sup _{t_{0}+n-1 \leq t \leq t_{0}+n}|x(t)|^{2}\right) \leq \beta e^{\gamma n}
$$

where $\beta=\frac{k_{0}}{1-k_{0}} E\|\xi\|^{2}+\frac{2\left[3+2 \pi(U)+2 \tilde{c}_{2}^{2}\right] L\left(T-t_{0}\right)}{\left(1-k_{0}\right)^{2}}$ and $\gamma=\frac{2 L\left[\left(1+k_{0}^{2}\right)+3+2 \pi(U)+2 \tilde{c}_{2}^{2}\right]}{\left(1-k_{0}\right)^{2}}$. Hence, for any $\varepsilon>0$, by the chebysher inequality, it follows that

$$
P\left\{\omega: \sup _{t_{0}+n-1 \leq t \leq t_{0}+n}|x(t)|^{2}>e^{(\gamma+\epsilon) n}\right\} \leq \beta e^{-\epsilon n} .
$$

Since $\Sigma_{n=0}^{\infty} \beta e^{-\varepsilon n}<\infty$, by the Borel-Cantelli lemma, we deduce that, there exists a integer $n_{0}$ such that

$$
\sup _{t_{0}+n-1 \leq t \leq t_{0}+n}|x(t)|^{2} \leq e^{(\gamma+\varepsilon) n} \quad \text { a.s } n \geq n_{0} .
$$

Thus, for almost all $\omega \in \Omega$, if $t_{0}+n-1 \leq t \leq t_{0}+n$ and $n \geq n_{0}$, then

$$
\frac{1}{t} \log |x(t)|=\frac{1}{2 t} \log \left(|x(t)|^{2}\right) \leq \frac{(\gamma+\varepsilon) n}{2\left(t_{0}+n-1\right)} .
$$

Taking limsup in (85) leads to almost surely exponential estimate, that is,

$$
\limsup _{t \rightarrow \infty} \frac{1}{t} \log |x(t)| \leq \frac{\gamma+\varepsilon}{2}=\frac{L\left[\left(1+k_{0}^{2}\right)+3+2 \pi(U)+2 \tilde{c}_{2}^{2}\right]}{\left(1-k_{0}\right)^{2}}, \quad \text { a.s. }
$$

Required assertion (84) follows because $\varepsilon>0$ is arbitrary.

\section{Conclusion}

In this paper, we prove the existence and uniqueness of the solution to NSFDEs with pure jumps under the Local Lipschitz condition. Meanwhile, by using the Itô formula, Taylor formula and the Burkholder-Davis inequality, we establish the $p$-th exponential estimations and almost surely asymptotic estimations of the solution to NSFDEs with pure jumps. 


\section{Acknowledgements}

The authors would like to thank the Royal Society of Edinburgh, Qing Lan Project of Jiangsu Province(2012), NNSF of China (11071037,11401261,61374080), NSF of Higher Education Institutions of Jiangsu Province (13KJB110005), the grant of Jiangsu Second Normal University(JSNU-ZY-02) and the Jiangsu Government Overseas Study Scholarship for their financial support.

\section{References}

[1] S. E. A. Mohammed, Stochastic Functional Differential Equations, Longman Scientific and Technical, 1984.

[2] X. Mao, Stochastic Differential Equations and Applications,Horwood, New York, 1997.

[3] X. Mao, Razumikhin-type theorems on exponential stability of stochastic functional differential equations, Stoch. Processes. Appl, 65 (1996), 233-250.

[4] X. Mao, A note on the LaSalle-type theorems for stochastic differential delay equations, J. Math. Anal. Appl. 268 (2002), 125-142.

[5] X. Mao, M. Rassias, Khasminskii-type theorems for stochastic differential delay equations, J. Sto. Anal. Appl, 23 (2005), 1045-1069.

[6] E. Buckwar, Introduction to the numerical analysis of stochastic delay differential equations, J. Comput. Appl. Math, 125 (2000), 297-307.

[7] U. Kuchler, E. Platen, Strong discrete time approximation of stochastic differential equations with time delay, Math. Comput. Simulation, 54 (2000), 189-205.

[8] Y. Hu, S. E. A. Mohammed, F. Yan, Discrete-time approximation of stochastic delay equations: the Milstein scheme, Ann. Probab, 32 (2004), 265-314.

[9] X. Mao, Exponential stability of equidistant Euler-Maruyama approximations of stochastic differential delay equations, J.Comput.Appl.Math,200 (2007), 297-316.

[10] D. Xu, Z. Yang, Y. Huang, Existence-uniqueness and continuation theorems for stochastic functional differential equations,J. Differential Equations, 245 (2008), 1681-1703. 
[11] F. Wu, X. Mao and L. Szpruch, Almost sure exponential stability of numerical solutions for SDDEs, Numer. Math, 115 (2010), 681-697.

[12] J. Appleby, X. Mao and $\mathrm{H}$. Wu, On the almost sure running maxima of soluti ons of affine stochastic functional differential equations, SIAM J. Math. Anal, 42 (2010), 646-678.

[13] I. Gyongy, S. Sabanis, A Note on Euler Approximations for Stochastic Differential Equations with Delay, Appl. Math. Optimization, 68 (2013) 391-412.

[14] X. Mao, Razumikhin-type theorems on exponential stability of neutral stochastic functional differential equations, SIAM J. Math. Anal, 28 (1997), 389-401.

[15] X. Mao, Asymptotic properties of neutral stochastic differential delay equations, Stochastics and Stochastic Reports, 68 (2000), 273-295.

[16] V. Kolmanovskii, N. Koroleva, T. Maizenberg, X. Mao, A. Matasov, Neutral stochastic differential delay equations with Markovian switching. Stoch. Anal. Appl, 21 (2003), 819-847.

[17] Q. Luo, X. Mao, Y. Shen New criteria on exponential stability of neutral stochastic differential delay equations, Syst. Control. Lett, 55 (2006), 826834.

[18] L. Huang, F. Deng, Razumikhin-Type Theorems on Stability of Neutral Stochastic Functional Differential Equations, IEEE Transactions on Automatic Control, 53 (2008) 1718-1723.

[19] H. Bao, J. Cao, Existence and uniqueness of solutions to neutral stochastic functional differential equations with infinite delay, Appl. Math. Comput, 215 (2009), 1732-1743.

[20] F. Wu, S. Hu, C. Huang Robustness of general decay stability of nonlinear neutral stochastic functional differential equations with infinite delay, Syst. Control. Lett, 59 (2010), 195-202.

[21] F. Wu, X. Mao, Numerical Solutions of Neutral Stochastic Functional Differential Equations, SIAM J. Numer. Anal, 46 (2008), 1821-1841.

[22] S. Jankovic, M.Vasilova, M.Krstic, Some analytic approximations for neutral stochastic functional differential equations, Appl. Math. Comput, 217 (2010) 3615-3623. 
[23] W. Wang, Y. Chen, Mean-square stability of semi-implicit Euler method for nonlinear neutral stochastic delay differential equations, Appl. Numer. Math, 61 (2011), 696-701 .

[24] S. Zhou, Z. Fang, Numerical approximation of nonlinear neutral stochastic functional differential equations, J. Appl. Math. Comput, 41 (2013), 427-445.

[25] Z. Yu, The improved stability analysis of the backward Euler method for neutral stochastic delay differential equations, I. J. Comput. Math, 90 (2013), 1489-1494.

[26] N. Ikeda, S. Watanabe, Stochastic Differential Equations and Diffusion Processes, North Holland Publishing Company, Amsterdam, Oxford, New York, 1989.

[27] H. Kunita, Stochastic diffrential equations based on Levy processes and stochastic flows of diffomorphisms, in Real and Stochastic Analysis, New Perspectives, M.M.Rao (ed.), 305-373, Birkhauser Boston Basel Berlin (2004).

[28] D. Applebaum, Levy Processes and Stochastic Calculus, Cambridge University Press (2004), second edition (2009)

[29] P. Billingsley, Convergence of probability measures, JohnWiley and Sons, Inc., New York-London- Sydney 1968. 